\title{
Fingerprint Recognition Using Markov Chain and Kernel Smoothing Technique with Generalized Regression Neural Network and Adaptive Resonance Theory with Mapping
}

\author{
Hemad Heidari Jobaneh \\ Department of Electrical Engineering, Azad University, South Tehran Branch, Tehran, Iran \\ Email address: \\ emehhj@gmail.com \\ To cite this article: \\ Hemad Heidari Jobaneh. Fingerprint Recognition Using Markov Chain and Kernel Smoothing Technique with Generalized Regression \\ Neural Network and Adaptive Resonance Theory with Mapping. Machine Learning Research. Vol. 4, No. 1, 2019, pp. 7-12. \\ doi: $10.11648 /$ j.mlr.20190401.12
}

Received: April 4, 2019; Accepted: May 16, 2019; Published: June 4, 2019

\begin{abstract}
The necessity of fast and precise identification from fingerprints might be fulfilled via systems benefiting from intelligent elements such as Neural Networks. The process of recognition and classification have been performed according to beneficial points called core point, singularities, or minutiae. However, points always are sensitive to noise and distortion, thus inaccurate results. Hence, instead of extracting a point, two lines are defined to bring down the risk of finding a point. Plus, two approaches are proposed with the intention of extracting statistical features predicated upon Kernel and Markov chain. In fact, two sets of features are extracted from both horizontal and vertical Markov chain, derived from the ridges angle around the aforementioned lines. In addition, all features are trained and tested via two divergent neural networks, consisting Generalized Regression Neural Network (GRNN) and Adaptive Resonance Theory with mapping (ARTMAP). Fingerprint verification competition (FVC) database is used to analyze the system. The performances of networks with different sets of features are simulated and compared with MATLAB. The results coming from simulation are compared and $93.5 \%$ and $83.5 \%$ accuracy is achieved for GRNN and ARTMAP respectively. Furthermore, the system is tested by both networks with features coming from just vertical and horizontal features.
\end{abstract}

Keywords: ARTMAP, Fingerprint Recognition, GRNN, Kernel, Markov Chain, Neural Network

\section{Introduction}

Fingerprints can be classified in five categories called whorl, right loop, left loop, arch, and tented arch via Henry system [1]. Owing to the similarity between arch and tended arch, they can be considered as one category, leading up to four categories, demonstrated in figure 1.

Fingerprints are composed of Ridges, Bifurcations, and Valleys, depicted in figure 2. The quality, direction, and the position of these elements are decisive factors by which fingerprints can be classified or identified.

Procedures like enhancement, feature extraction, and classifiers are common in both classification and recognition. Gabor filter is one of the most beneficial methods used to enhance ridges quality [2]. In addition, the core point is used to extract a point around which fingerprints with same class behave similarly [3-4]. Hence, features extracted around the core point are movement invariant. In addition, Singularities comprising of both core and delta point are other useful points to facilitate recognition [5-7].

Because of the rotation of images, features might be influenced considerably. Therefore, an approach is proposed to rectify the problem [8]. Ridges Orientation might be considered as an advantageous method with the intention of feature extraction [9].

Recognition process has been performed by many methods. Fuzzy system is used for classification and feature extraction [10-12]. Artificial Neural Network (ANN) is picked out to categorize fingerprints [13-14]. Decision trees and Markov models are combined to classify fingerprints by the ridge structure of the fingerprints [15].

In this paper, an approach is proposed to extract two lines around which the behavior of ridges and valleys are obtained as a part of final features. Plus, methods including Kernel 
smoothing technique, Markov chain, and angular behavior of Ridges and Valleys are used to extract fingerprints features. Generalized regression neural network (GRNN) and Adaptive Resonance Theory with mapping (ARTMAP) are used and compared for the sake of recognition.

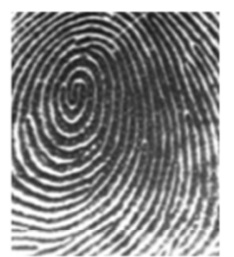

(a)

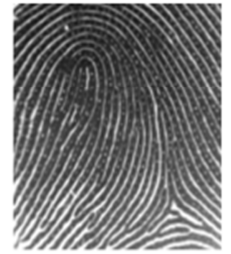

(c)

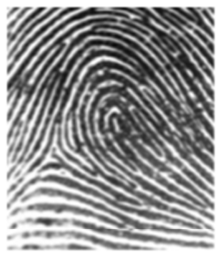

(b)

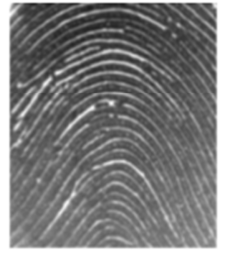

(d)
Figure 1. Fingerprints categories: (a) whorl, (b) right loop, (c) left loop, (d) arch.

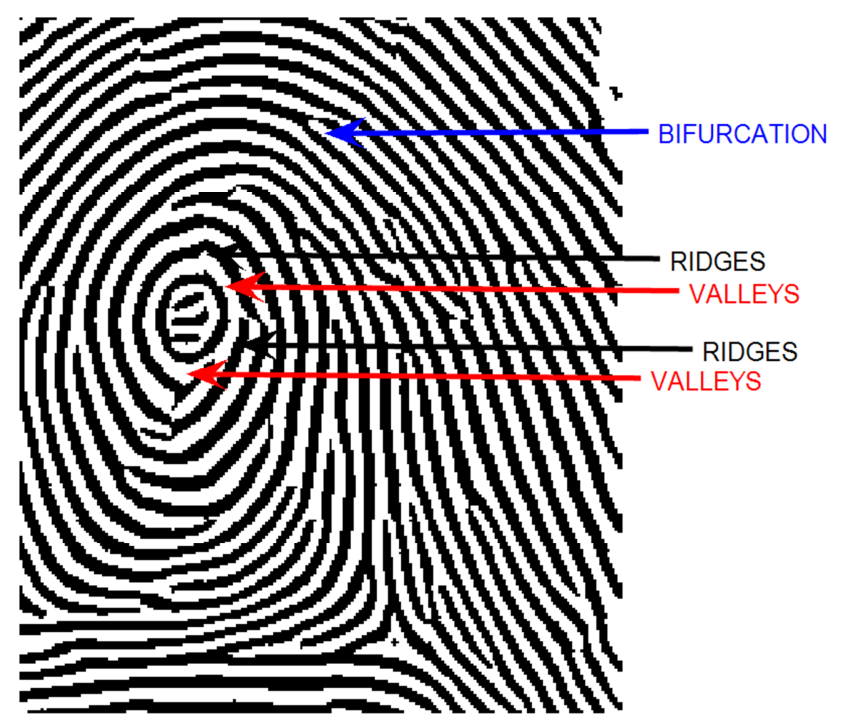

Figure 2. Ridges, Valleys, and Bifurcation.

\section{Vertical and Horizontal Lines}

Minutiae points, which include ridge ending and bifurcation points, are reliable factors which have been used in recognition procedure [16-18]. Nonetheless, unavoidable distortion might exacerbate the process dramatically. For instance, a bifurcation can be converted to a short or long ridge by destroying the junction of Bifurcation, illustrated in figure 3. Therefore, features should be predicated upon factors which are less dependent on points.

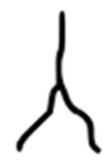

(a)

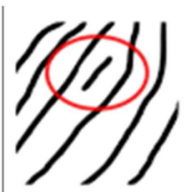

(c)

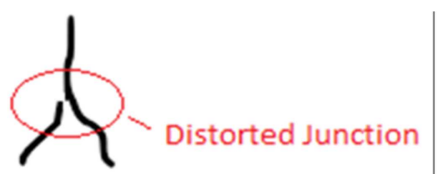

(b)

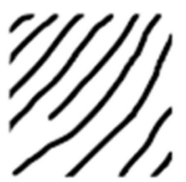

(d)
Figure 3. (a) Bifurcation (b) Distorted Bifurcation (c) Short Ridge (d) Ridge Ending.

To commence the process, two steps should be performed, consisting of the orientation of ridges and enhancement [19]. In order to extract the orientation of ridges, the gradient of enhanced images is calculated, shown in figure 4. In fact, images can be taken into account as $f(x, y)$ which its gradient can be calculated by:

$$
\nabla f(x, y)=\frac{\partial f}{\partial x} \hat{i}+\frac{\partial f}{\partial y} \hat{j}
$$

Plus, if $D x=\frac{\partial f}{\partial x}$ and $D y=\frac{\partial f}{\partial y}$, then the direction of ridges is achieved by:

$$
D=\tan ^{-1}\left(\frac{D y}{D x}\right)+\frac{\pi}{2}
$$

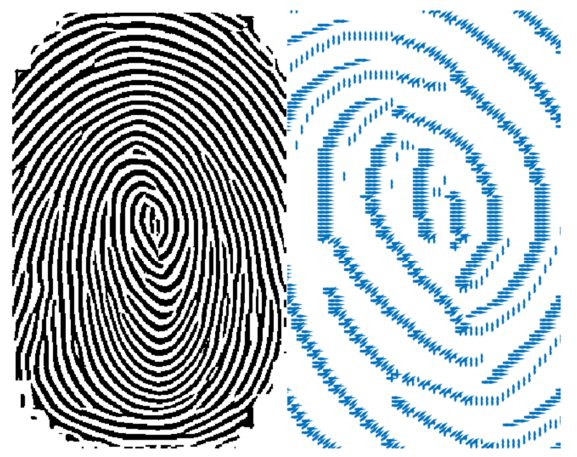

(a)

(b)

Figure 4. (a) Enhanced image (b) Gradient.

Part of fingerprints might be ruined, thus the wrong classification and recognition. Therefore, images should be divided into meaningful parts. In other words, each part of an image should be compared with its counterpart in other pictures. To clarify, once the direction of ridges is obtained, images are split into two parts, including acute-angles and obtuse angles, demonstrated in figure 5. Next, the features extracted from acute angles are compared with corresponding parts of other pictures.

Points are critical and sensitive elements for the process. Shown in figure 3, points are contingent upon the quality of 
pictures and with any noise or distortion they might be calculated inaccurately. Hence, to find a location as the origin of beginning the process, two lines are considered as the origin areas, demonstrated in figure 6.

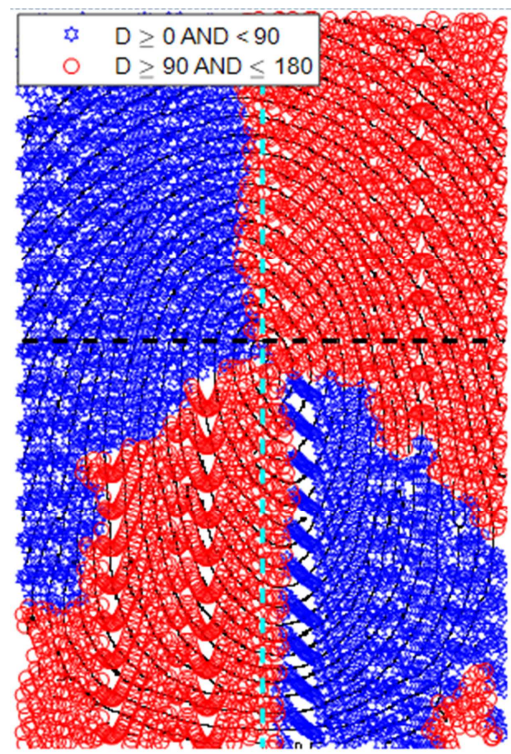

Figure 5. Divided into two parts.

The vertical line should fulfil the following criteria:

$$
\left\{\begin{array}{l}
D \geq 170^{\circ} \\
\frac{\partial D}{\partial y} \neq 0 \\
\frac{\partial D}{\partial x}=0
\end{array}\right.
$$

First criterion indicates that the vertical line crosses from or near ridges with angles more than one hundred seventy degrees. The second condition is proposed in order to eliminate false lines around the corner of fingerprints, depicted in figure 7.

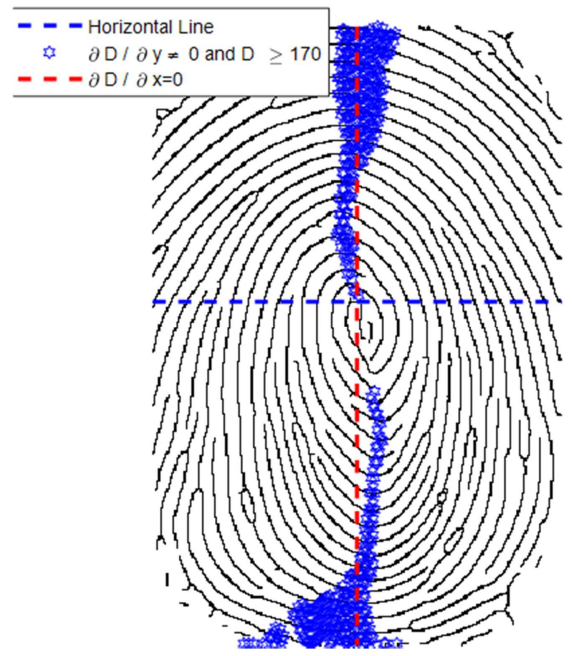

Figure 6. Horizontal and Vertical Lines.
In fact, around the corner of fingerprints a white area exists and creates valleys with one hundred eighty degrees, which fulfil the first condition ( $\mathrm{D}>170)$. However, the second condition eliminates this part.

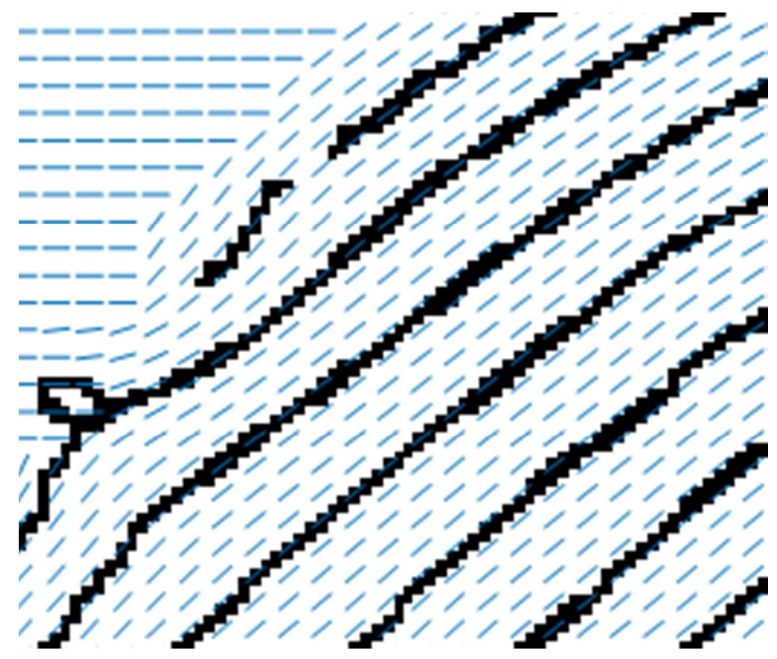

Figure 7. False lines around the corner.

In addition, the third condition defines the ridges which have $\mathrm{D}>170$ and vertically have the same angles. As it is shown in figure 6 , it might be more than one object conforming to the criteria. In this case, the upper object is the main object and the tangent line to it is the horizontal line.

\section{Feature Extraction and Vertical and Horizontal Markov Chain}

The training vector is defined:

Training and Test vector $=[$ Kernel of cropped block from the point (18 numbers), Horizontal Markov chain coefficients extracted from ridges with acute angles (12 numbers), Horizontal Markov chain coefficients extracted from ridges with obtuse angles (12 numbers), Vertical Markov chain coefficients extracted from ridges with acute angles (30 numbers), Vertical Markov chain coefficients extracted from ridges with obtuse angles (30 numbers)]

In fact, after finding the vertical and horizontal lines, the intersection of these lines is the coordinates around which an $n \times m$ block is cropped. Next, Kernel smoothing technique is applied to the block to extract part of training vector, demonstrated in figure 8 . The smoothing formula is given by:

$$
\hat{f}_{h}(x)=\frac{1}{n h} \sum_{i=1}^{n}\left(\frac{x-x_{i}}{h}\right) \quad ;-\infty<x<\infty
$$

In which $\mathrm{K}$ is the kernel smoothing function, $h$ is bandwidth, and $\mathrm{n}$ is the sample size. These features demonstrate general characteristics of fingerprints in different kinds of fingerprints according to Henry system. 


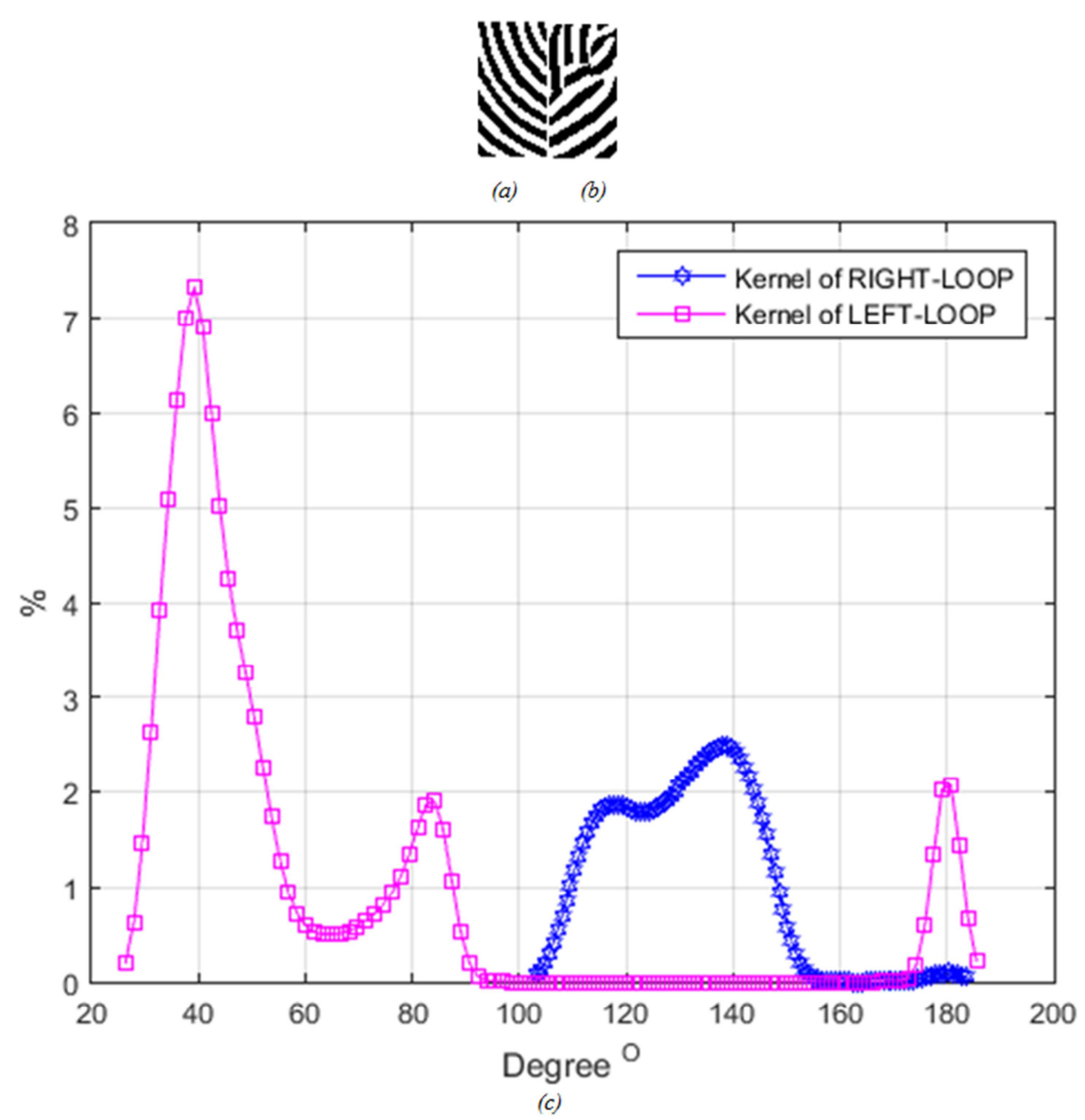

Figure 8. (a) Cropped image of RIGHT-LOOP (b) Cropped image of LEFT-LOOP (c) Kernel-RIGHT-LOOP and Kernel-LEFT-LOOP.

Once an image is divided into two parts including acute and obtuse angles, the vertical and horizontal lines are applied to both pictures and according to the location of lines and the size of the picture, the image is divided into equal blocks. In fact, each block constitutes a state of Markov chain, depicted in figure 9. Markov chain is a series of states in which each state depends on previous state so that transition matrix is the changes of states which can be achieved by following method [20]:

$$
\begin{aligned}
p\left[q_{t}\right. & \left.=s_{j} \mid q_{t-1}=s_{i}, q_{t-2}=s_{k}, \ldots\right] \\
& =p\left[q_{t}=s_{j} \mid q_{t-1}=s_{i}\right]
\end{aligned}
$$

And the state transition coefficients have the properties:

$$
a_{i j}=p\left[q_{t}=s_{j} \mid q_{t-1}=s_{i}\right], \quad 1 \leq i, j \leq N
$$

$\mathrm{N}$ is the number of states. These coefficients should define the circumstances of equation 7 [20].

$$
a_{i j} \geq 0, \sum_{j=1}^{N} a_{i j}=1
$$

Next, in order to extract more specific features, each image is modeled as two vertical and horizontal Markov chains, demonstrated in figure 10. In this case, $a_{i j}=0$ for $i=j$. The percentage of alteration between each corresponding ridge in each state generates a coefficient between two states, demonstrated in figure 11.

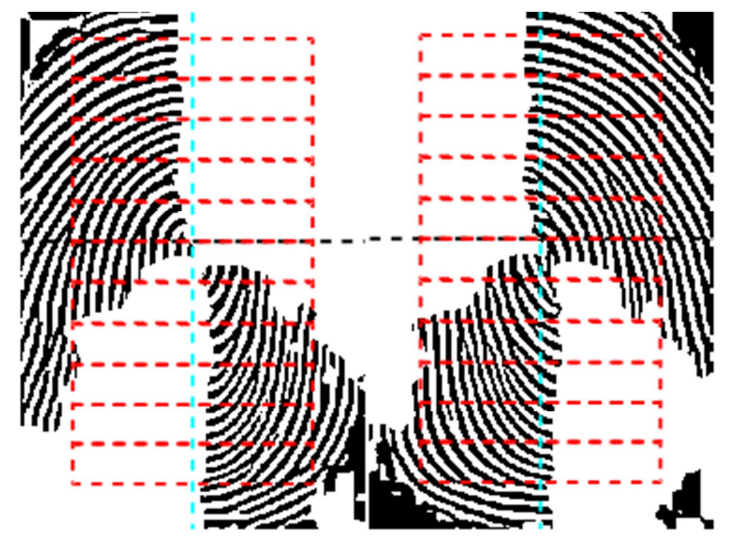

(a)

(b)

Figure 9. Vertical and Horizontal states of Markov Chain in (a) Acute angles (b) Obtuse angles. 


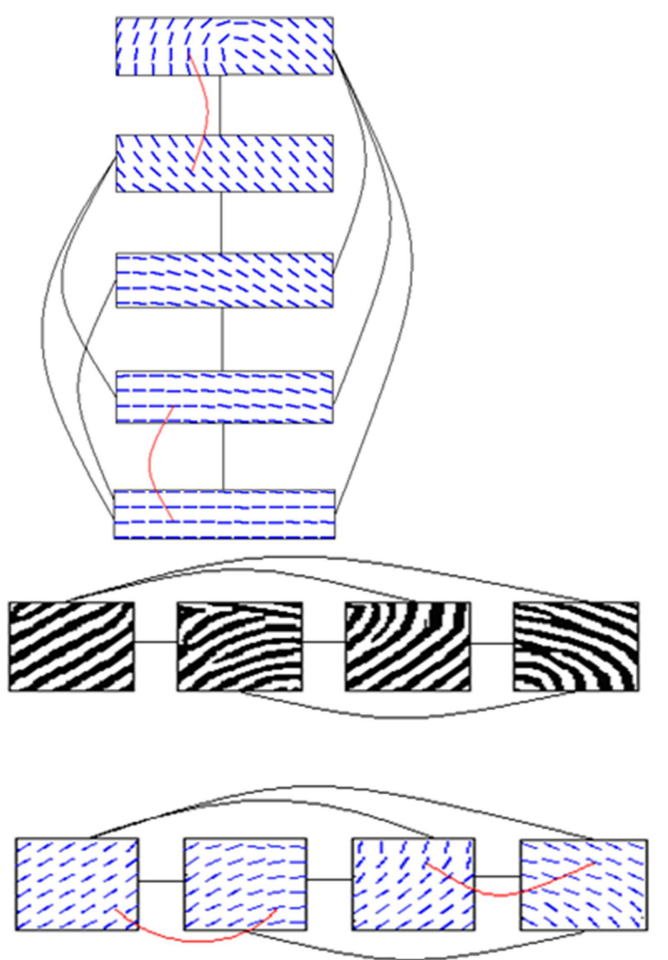

Figure 10. Vertical and Horizontal states of Markov Chain
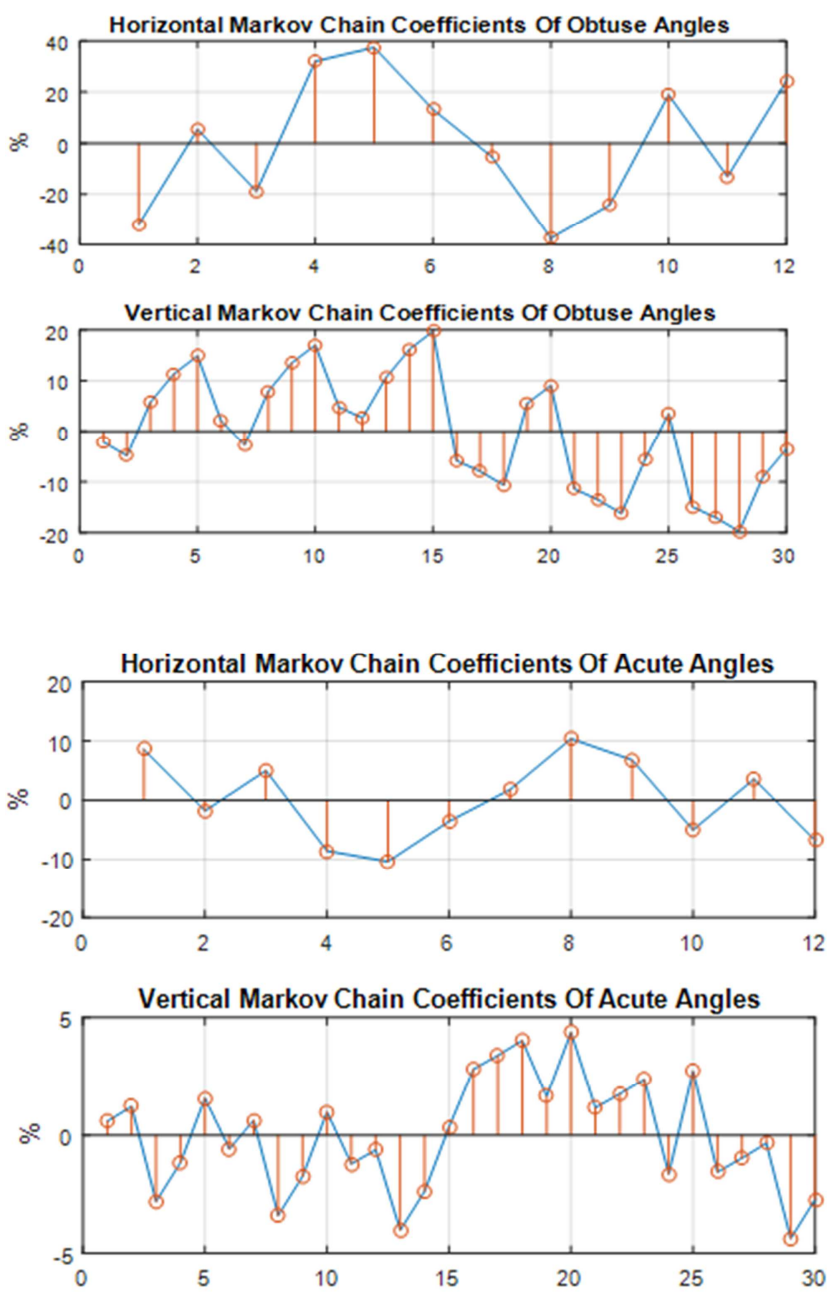

Figure 11. Vertical and Horizontal Markov Chain coefficients.

\section{GRNN and ARTMAP Neural Networks}

The models of ARTMAP were described by Carpenter in 1992. The network is compounded of two supervised and unsupervised neural networks [21]. The network is learnt by match-based learning method. This method is good enough for online learning and big databases. Vigilance parameter is one of the most significant parameters of ARTMAP. This parameter is set 0.91 in this work.

GRNN is kind of radial basis network with a linear layer in the second layer. It is suggested that GRNN be used to approximate or classify data or functions [22]. The spread parameter is a decisive factor in designing the network. It affects the performance of the network significantly. The designed network is illustrated in figure 12. Each image includes one hundred two figures. Plus, the spread is 0.4 for GRNN. FVC2004 DB1_A is used to train and test both networks [23]. Two hundred of images are used to test the network. The simulation is performed by MATLAB. The performances of both networks are compared in table1.

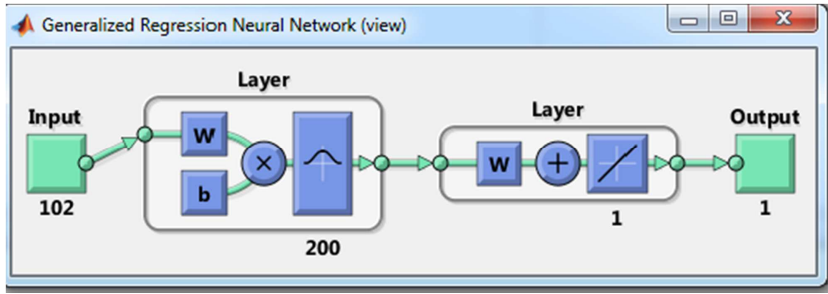

Figure 12. Designed GRNN.

In fact, the performances of both networks are evaluated with different sets of features separately. The best result is achieved with the participation of all features and GRNN. Plus, the worst result is obtained with GRNN and just Kernel technique.

Table 1. Results of Simulation.

\begin{tabular}{ll}
\hline Method & Accuracy (\%) \\
\hline ARTMAP+Kernel & 56 \\
ARTMAP+HORIZONTAL COEFFICIENTS & 62 \\
ARTMAP+VERTICAL COEFFICIENTS & 65 \\
ARTMAP+VERTICAL AND HORIZONTAL & 78.5 \\
COEFFICIENTS & \\
ARTMAP+Kernel+HORIZONTAL & 88.5 \\
COEFFICIENTS+VERTICAL COEFFICIENTS & 48.5 \\
GRNN+Kernel & 53 \\
GRNN+HORIZONTAL COEFFICIENTS & 47 \\
GRNN+VERTICAL COEFFICIENTS & 69.5 \\
GRNN+VERTICAL AND HORIZONTAL & \\
COEFFICIENTS & 93.5 \\
GRNN+Kernel+HORIZONTAL & \\
COEFFICIENTS+VERTICAL COEFFICIENTS & \\
\hline
\end{tabular}

In some cases, fingerprints are recognized incorrectly. Nevertheless, the system demonstrates that they are similar to an image in the same class. It implies that even though the features are not appropriate for recognition, they might be beneficial for classification. 


\section{Conclusion}

An approach for recognition is proposed in this paper. In feature extraction, statistical methods, including Markov chain and Kernel distribution are used. Fuzzy system is a powerful system which has been used in the fingerprint identification process [10-12]. One of the most noticeable advantages of neural networks over fuzzy is the rule extraction. In other words, the rules in fuzzy have to be defined by experts in the specific fields. Neural networks, on the other hands, extract rules according to their algorithm. Hence, the recognition process is carried out by two divergent neural networks consisting of ARTMAP and GRNN. The performance of GRNN outweighs ARTMAP. However, when it comes to test vector with less elements the performance of ARTMAP is better. Two hundred of images are used in the training phase and two hundred of them are used in test phase.

\section{References}

[1] E. R. Henry. "Classification and Uses of Fingerprints," HM Stationery Office, 1905.

[2] C. J. Lee, S. D. Wang, "Fingerprint feature reduction by principal Gabor basis function," Pattern Recognition, 34, 2001, pp. 2245-2248.

[3] C. Park, S. Oh, D. Kwak, B. Kim, Y Song, and K Park, "A new reference point detection algorithm based on orientation pattern labeling in fingerprint images", pp. 697-703, Pattern Recognition and Image Analysis, First Iberian Conference, IbPRIA 2003.

[4] B. Bhanu and X. Tan, Fingerprint indexing based on novel features of minutiae triplets, IEEE Trans. PAMI, May 2003.

[5] L. Wei, "Fingerprint Classification using Singularities Detection", International Journal of Mathematics and computers in simulation, Vol. 2, No. 2, pp. 158-162, 2008.

[6] Iwasokun, Gabriel Babatunde, and O. C. Akinyokun. "Fingerprint Singular Point Detection Based on Modified Poincare Index Method." International Journal of Signal Processing Image Processing \& Pattern Recognition 7, 2014.

[7] M. Liu. "Fingerprint Classification Based on Singularities," Pattern Recognition, Nov. 2009, pp. 1-5. doi: 10.1109/CCPR.2009.5343966.

[8] P. Gnanasivam and S. Muttan, "An efficient algorithm for fingerprint preprocessing and feature extraction”, ICEBT 2010, Procedia computer Science, Vol. 2, 2010, pp. 133-142.

[9] S. C. Dass, A. K. Jain. "Fingerprint Classification Using Orientation Field Flow Curves," Proc. Proceedings of the Fourth Indian Conference on Computer Vision, Graphics \& Image Processing, Dec. 2004, pp. 650-655.

[10] S. M. Mohamed and H. O. Nyongesa, "Automatic Fingerprint Classification System Using Fuzzy Neural Techniques", proc.
Of the IEEE International Conference on Fuzzy System, 2002, Vol. 1, pp. 358-362.

[11] K. Nandakumar, A. K. Jain, and S. Pankanti, "Fingerprintbased Fuzzy Vault: Implementation and Performance," IEEE Trans. on Info. Forensics and Security, vol. 2, no. 4, pp. 744 757, December 2007.

[12] Surmacz, K., Saeed, K., Rapta, P., “An improved algorithm for feature extraction from a fingerprint fuzzy image", Optica Applicata, Volume 43 - No. 3, 2013, Pages 515 527.

[13] S. R. Patil and S. R. Suralkar, "Fingerprint Classification using Artificial Neural Network", International Journal of Emerging Technology and Advanced Engineering, Volume 2, Issue 10, pp. 513-517, 2012, ISSN 2250-2459.

[14] V. Conti, C. Militello, S. Vitabile and F. Sorbello, "An Embedded Fingerprints Classification System based on Weightless Neural Networks", Frontiers in Artificial Intelligence and Applications - IOS Press Editor, Volume 193: New Directions in Neural Networks, 2009, pp. 67-75, ISSN 0922-6389, doi: 10.3233/978-1-58603-984-4-67.

[15] A. Senior, "A Combination Fingerprint Classifier", IEEE Transaction on Pattern Analysis and Machine Intelligence, 2001, Vol. 23, No. 10, pp. 1165-1174.

[16] Bhanu, B., Tan, X.: Fingerprint indexing based on novel features of minutiae triplets. IEEE Trans. Pattern Anal. Mach. Intell. 25 (5), 616-622 (2003).

[17] H. Fronthaler, K. Kollreider, and J. Bigun. Local features for enhancement and minutiae extraction in fingerprints. Image Processing, IEEE Trans. on, 17 (3): 354 -363, march 2008.

[18] Sankaran A, Pandey P, Vatsa M, et al. On latent fingerprint minutiae extraction using stacked denoising sparse auto encoders [C]// Biometrics (IJCB), 2014 IEEE International Joint Conference on. IEEE, 2014: 1-7.

[19] R. Thai. (2003). Fingerprint Image Enhancement and Minutiae Extraction. The University of Western Australia. Retrieved from http://www.peterkovesi.com/studentprojects/raymondthai/Ray mondThai.pdf.

[20] Fort, A., Mugnaini, M. and Vignoli, V. Hidden Markov Models Approach used for Life Parameters Estimations. Reliability Engineering and System Safety, vol. 136, pp. 85-91. 2015.

[21] Gail A. Carpenter \&Stephen Grossberg, "ADAPTIVE RESONANCE THEORY", 1998.

[22] A. J. Al-Mahasneh, S. G. Anavatti, and M. A. Garratt, "Altitude identification and intelligent control of a flapping wing micro aerial vehicle using modified generalized regression neural networks," in 2017 IEEE Symposium Series on Computational Intelligence (SSCI). IEEE, 2017, pp. 23022307.

[23] FVC2004 Fingerprint Verification Competition http://bias.csr.unibo.it/fvc2004 download.asp. 